\title{
UV cross-linking/immunoprecipitation assay: glucocorticoid receptor-adrenergic receptor gene sequence interaction
}

\author{
Ying Bai ${ }^{1}$, Philbert Kirigiti ${ }^{1}$, Xiaorong Li ${ }^{1}$, Biao $\mathrm{Li}^{2}$, Li Tian${ }^{1}$, \\ Mark Y.-J. Ma², and Curtis A. Machida ${ }^{1}$
}

BioTechniques 35:100-111 (July 2003)

\begin{abstract}
The rat $\beta_{1}$-adrenergic receptor ( $\beta_{1}$-AR) gene contains glucocorticoid response element (GRE) half-sites at positions -2767 and -945. In electrophoretic mobility shift assay (EMSA) experiments, neither $\beta_{1}$-AR GRE half-site recognized glucocorticoid receptors (GRs) obtained from baculovirus high-level expression systems or from mammalian cells. We have developed a sensitive UV crosslinking/immunoprecipitation assay, using a 524-bp fragment containing the prototypical GRE obtained from the rat tyrosine aminotransferase promoter sequence and using antibodies recognizing mammalian GR. Using this assay, we provide evidence that rat $\beta_{1}$-AR gene sequences recognize mammalian GRs expressed in mouse $3 T 3$ cells and that the site of GR interaction does not appear to specifically contain the $\beta_{1}$-AR GRE half-sites. This represents one of the first reports demonstrating the utility of a UV cross-linking/immunoprecipitation assay in the detection of mammalian GR interaction with $\beta_{1}$-AR sequences, is consistent with the lack of specific DNA-GR protein complexes observed in EMSA experiments using oligonucleotide probes containing the $\beta_{1}$-AR GRE half-sites, and provides evidence that mammalian GR interaction occurs at complex rat $\beta_{1}$-AR gene sequences.
\end{abstract}

\section{INTRODUCTION}

Glucocorticoids can serve as direct regulators of $\beta$-adrenergic receptor $(\beta$ AR) gene transcription (1-3). Treatment of DDT ${ }_{1} \mathrm{MF}-2$ muscle cells with glucocorticoids results in the rapid elevation of $\beta_{2}$-AR mRNA and receptor levels (1). In addition, in other established cell lines, including the C6 glioma line, glucocorticoid treatment results in either the repression or induction of $\beta_{1}$-AR and $\beta_{2}$-AR mRNA transcription, respectively $(3,4)$. Dexamethasone treatment of 3T3-L1 cells, which express both $\beta_{1}$-ARs and $\beta_{2}$ ARs, also resulted in differential regulation of $\beta$-AR subtype mRNAs (5). Furthermore, in $\mathrm{DDT}_{1} \mathrm{MF}-2$ cells, dexamethasone treatment can reverse agonist-induced down-regulation of $\beta_{2}$-AR mRNA and receptor levels $(5,6)$.

Consensus matches of putative glucocorticoid response elements (GREs) (5'-GGTACANNNTGTTCT-3') have been identified within the human $\beta_{2}$ AR gene at positions -248 and -1447 (6). Prototypical GREs are composed of two hexameric half-sites (5'-GGTACA-3') that form a palindrome separated by a 3-base spacer (7). Each GRE half-site interacts with one glucocorticoid receptor (GR) monomer; GRmediated transcriptional activation is initiated by GR homodimer or heterodimer interaction with a complete GRE site. In additional studies, six potential GREs in the rat $\beta_{2}$-AR gene have been identified; the use of $\beta_{2}$-AR promoter-luciferase recombinants indicates that only one of these GREs, located at positions -379 to -365 , is necessary for glucocorticoid-dependent stimulation of receptor gene expression (8). Two potential GRE halfsites have been identified at positions -2767 and -945 in the rat $\beta_{1}$-AR gene (Reference 9 and unpublished observations); the 25-bp region between nucleotide positions -950 and -926 has been reported to be responsive to glucocorticoid-induced transcriptional repression using $\beta_{1}$-AR promoter-luciferase recombinants in $\mathrm{C} 6$ transfectant cells and appears to recognize rabbit reticulolysate-generated GRs (9). The $\beta_{1}$-AR GRE half-sites do not appear to constitute the complete palindromic GRE sequence, typically noted as positive GREs for their up-regulation effect on gene transcription. In addition, a novel glucocorticoid regulatory unit (GRU) that conveys positive glucocorticoid responsiveness has been identified in the ovine $\beta_{1}$-AR gene; this 43-bp region, extending from positions -1274 to -1232 , contains a core binding motif for homeodomain proteins, an E-box binding site for the Myc/Max family proteins, and an overlapping GRE half-site (10).

In this report, we wanted to verify the interaction of mammalian-derived GR protein in electrophoretic mobility shift assays (EMSAs), using the $\beta_{1}$-AR 
sequence surrounding the GRE half-site at positions -2767 and -945 . The 25 -bp sequence between positions -950 and -926 of the rat $\beta_{1}$-AR gene, described to interact with rabbit reticulolysate-generated GRs, did not recognize GR obtained from baculovirus expression systems or from mammalian cells. Using a sensitive UV cross-linking/immunoprecipitation assay, we provide evidence that rat $\beta_{1}$-AR gene sequences recognize mammalian GRs expressed in mouse 3T3 cells and that the site of GR interaction is complex and does not appear to specifically contain the $\beta_{1}$-AR GRE half-sites. This represents one of the first reports to demonstrate the utility of a sensitive UV cross-linking/immunoprecipitation assay in the detection of mammalian GR interaction with $\beta_{1}$-AR sequences.

\section{MATERIALS AND METHODS}

\section{Cell Lines and Sequence Analysis Programs}

The monkey kidney CV-1 (ATCC CCL70) and mouse 3T3 (ATCC CRL 6588) cell lines were cultured in DMEM, supplemented with $2.5 \%$ heatinactivated fetal calf serum (FCS), $7.5 \%$ calf serum, $100 \mathrm{U} / \mathrm{L}$ penicillin, 1 $\mathrm{mg} / \mathrm{L}$ streptomycin, and $2.5 \mathrm{mM}$ L-glutamine. Automated DNA sequencing support was provided by the Molecular \& Cell Biology Core Facilities at the Oregon Health and Science University. Auxillary in-laboratory sequence analyses of $\beta_{1}$-AR sequences were conducted with the use of the MacVector 5.0 program (Eastman Kodak, Rochester, NY, USA).

\section{Oligonucleotide Probes and Primers}

Oligonucleotide DNA probes for EMSA were synthesized from SigmaGenosys (The Woodlands, TX, USA). For EMSA analyses of GREs, sense oligonucleotides and reverse complement oligonucleotides were synthesized and annealed at equal molar concentrations before use. The annealed double-stranded oligonucleotides were electrophoresed in $6 \%$ nondenaturing polyacrylamide gels, eluted in a buffer consisting of $10 \mathrm{mM}$ Tris- $\mathrm{HCl}, \mathrm{pH} 8.0$,
$1 \mathrm{mM}$ EDTA, and $0.1 \mathrm{mM} \mathrm{NaCl}$, subjected to several cycles of freeze $\left(-85^{\circ} \mathrm{C}\right)$ and thaw $\left(37^{\circ} \mathrm{C}\right)$, and then purified by organic extractions and ethanol precipitation. End-labeled probes, including PCR fragments and annealed oligonucleotides, were purified using Sephadex ${ }^{\circledR}$ G-50 columns and subjected to the Wizard ${ }^{\mathrm{TM}}$ DNA Clean Up Kit (Promega, Madison, WI, USA) before use in EMSA.

\section{GR Reagents}

The GR discovery toolkit, obtained from Affinity Bioreagents (Golden, CO, USA), contains (i) recombinant human GR expressed in a baculovirus/insect cell expression system and (ii) dsDNA containing the complete palindromic GRE (half-sites are underlined) from the rat tyrosine amino transferase (TAT) sequence (5'-CTAGGCTGTACAGGATGTTCTGCCTAG-3'). Polyclonal rabbit anti-glucocorticoid receptor $\alpha(\mathrm{GR} \alpha)$ antibody was also obtained from Affinity Bioreagents. In addition, we have also utilized the affinity-purified rabbit polyclonal antibody against the mouse GR $\alpha$ (raised against a peptide corresponding to amino acids 5-20; GR M-20; Santa Cruz Biotechnology, Santa Cruz, CA, USA). For GRE mutagenesis analyses, we obtained or synthesized four doublestranded oligonucleotides: (i) consensus GRE from Santa Cruz Biotechnology containing two half-sites (underlined) (5'-AGAGGATCTGTACAGGATGTTCTAGAT-3'); (ii) muta-genized GRE containing two ineffective half-sites with GT-to-CA substitutions in both half-sites (5'-AGAGGATCTCAACAGGATCATCTAGAT-3') [probe name: GRE (M1 and M2)]; (iii) mutagenized GRE containing intact upstream halfsite and scrambled downstream half-site (5'-AGAGGATCTGTACAGGACTGCAGAGAT-3') (probe name: GRE/ $\mathrm{M} 1$ ); and (iv) mutagenized GRE containing intact downstream half-site and scrambled upstream half-site $\left(5^{\prime}\right.$ AGAGGATCCTGCAGGGATGTTCTAGAT-3') (probe name: GRE/M2 probe). $\beta_{1}$-AR upstream sequence containing consensus GRE half-sites at positions -945 and -2767 were synthesized as EMSA probes: (i) $\beta_{1}$-AR sequence from -970 to -920 (reverse complement 3'-TCTCTCGTGTCGAGACGCGTT-
CTTGTCGCGGGTCTCTCCTCCGGAAAGACTGTCT-5') and (ii) $\beta_{1}$-AR sequence from -2793 to -2743 (sense complement 5'-ACTACTCAACCGCCCCTTCTGCTTTCTGTTCTGAAACACAGGCCCTCTTTA-3').

For the development of longer $\beta_{1}$ AR upstream probes containing the GRE half-sites and flanking DNA, we synthesized PCR probes internally labeled with $[\alpha-32$ P $]$ dCTP. For the development of the upstream $\beta_{1}$-AR probe (703-bp fragment), we synthesized primer A (5'-GCTCTAGAGCAGAGAGCCAGGTCATT-3'; positions -2844 to -2819) and primer B (5'-GGAATTCCTGGGATGATAAGGCC-3'; reverse complement; positions -2142 to -2164) For the development of the downstream $\beta_{1}$-AR probe (543-bp fragment), we synthesized primer $\mathrm{C}\left(5^{\prime}\right.$ GCTCTAGAGCCACCCTACCAAGA $-3^{\prime}$; positions -1350 to -1328$)$ and primer D (5'-GGAATTCCCTGATCGCCGAGGGA-3'; reverse complement; positions -808 to -830 ). Using primer pairs $\mathrm{A}-\mathrm{B}$ and $\mathrm{C}-\mathrm{D}, \mathrm{PCR}$ was conducted with the following reaction components: $1.5 \mathrm{mM} \mathrm{MgCl}_{2}, 0.2 \mathrm{mM}$ all deoxynucleotides except dCTP, $0.1 \mathrm{mM}$ dCTP, $0.1 \mathrm{mCi}\left[{ }^{32} \mathrm{P}\right] \mathrm{dCTP}, 250 \mathrm{ng}$ DNA template containing the rat $\beta_{1}$-AR gene sequence -3221 to $-1,100$ pmol each primer, and $5 \mathrm{U}$ Taq DNA polymerase. The reaction mixture was subjected to an initial denaturation at $94^{\circ} \mathrm{C}$ for $1 \mathrm{~min}$, followed by 35 cycles of $94^{\circ} \mathrm{C}$ for $1 \mathrm{~min}, 55^{\circ} \mathrm{C}$ for $45 \mathrm{~s}$, and $72^{\circ} \mathrm{C}$ for $1 \mathrm{~min}$. A final extension at $72^{\circ} \mathrm{C}$ for $1 \mathrm{~min}$ was performed, followed by soaking at $4^{\circ} \mathrm{C}$.

A similar PCR approach was also utilized in the development of a longer EMSA probe containing the complete palindromic GRE from the rat TAT gene (5'-CTAGGCTGTACAGGATGTTCTGCCTAG-3'; relative positions -2505 to -2491 in the rat TAT gene) flanked by additional DNA amplified from the endogeneous gene. A 524-bp genomic DNA fragment containing the TAT upstream region -2614 to -2091 was amplified by PCR using rat genomic DNA template and upstream (TAT/GRE oligonucleotide 1: 5'-AGGAATTCCTTCTCAGTGTTCTCTATC3'; EcoRI-engineered end) and downstream (TAT/GRE oligonucleotide 2: 5'-ACGGGATCCCGTCCTCATTCT- 
TCTTAATGC-3'; reverse complement; Bam HI-engineered end) primers. The 524-bp fragment was subcloned into the EcoRI and BamHI restriction endonuclease sites of pGEM $3 Z$, and the ensuing recombinant $\mathrm{pJ} 38$ was verified by sequence analyses.

\section{EMSA and UV Cross-Linking Procedures}

EMSA was performed using ${ }^{32} \mathrm{P}-\mathrm{la}-$ beled double-stranded oligonucleotide probes or PCR products. Nuclear extracts from cells were prepared as described in Kirigiti and Machida (11). Probes were incubated with nuclear extract in EMSA binding buffer (10 $\mathrm{mM}$ Tris- $\mathrm{HCl}, \mathrm{pH} 7.5,50 \mathrm{mM} \mathrm{NaCl}, 5$ $\mathrm{mM} \mathrm{MgCl} 2,1 \mathrm{mM}$ EDTA, and 5\% glycerol) for $25 \mathrm{~min}$ at room temperature $(11,12)$. In some cases, using methods described in Kirigiti et al. (12) and for the TAT GRE probe interaction, competition reactions were conducted using nonradioactive PCR fragments (the 524-bp DNA fragment containing the TAT upstream region) or double-stranded oligonucleotides containing the consensus TAT GRE (containing two effective half-sites), mutant TAT GRE [mutagenized GRE containing two ineffective half-sites with GT-to-CA substitutions in both half-sites; probe name: GRE (M1 and M2)], or nonrelevant sequences. For competitions using the PCR-generated $\beta_{1}$-AR upstream and downstream probes, we synthesized and prepared 30-bp oligonucleotide duplexes containing either the upstream GRE halfsite $\left(\beta_{1}\right.$-AR sequence from -2784 to -2755; half-site at position -2767) and downstream GRE half-site ( $\beta_{1}$-AR sequence from -961 to -932 ; half-site at position -945). As a nonrelevant sequence competitor, we used the 29-bp double-stranded oligonucleotide GS-1, encompassing the rat $\beta_{1}$-AR sequence -396 to -367 (12). In a prior publication (12), using a UV cross-linking/ immunoblot procedure, we demonstrated that the rat $\beta_{1}$-AR sequence -408 to -323 , which contains the GS-1 sequence as a subsequence, recognized one major protein of $117 \mathrm{kDa}$ and additional minor proteins of 37 and 35 $\mathrm{kDa}$; there were no UV cross-linked proteins that approached the $100-\mathrm{kDa}$ size observed for GRs. Competition reactions were based using $1 \times, 10 \times$, or $20 \times$ molar excess of nonradioactive DNA competitor. DNA-protein complexes were electrophoresed on $4 \%$ polyacrylamide gels in $1 \times$ Trisglycine-EDTA buffer $(50 \mathrm{mM}$ Tris base, $380 \mathrm{mM}$ glycine, $2 \mathrm{mM}$ EDTA, $\mathrm{pH}$ 8.8), and visualized by autoradiography (at $-85^{\circ} \mathrm{C}$ for $18-48 \mathrm{~h}$ ) using Kodak ${ }^{\circledR}$ M-5 film or by phosphorimager analysis with the Bio-Rad ${ }^{\circledR}$ Molecular Imager system (Bio-Rad Laboratories, Hercules, CA, USA). Antibodies used for supershift reactions include the polyclonal rabbit anti-GR $\alpha$ antibody obtained from Affinity Bioreagents.

For use in protein size determinations, the reaction mixtures were then placed on ice and subjected to UV irradiation for $5 \mathrm{~min}$ in a cross-linking oven (Stratalinker ${ }^{\circledR}$ oven; Stratagene, La Jolla, CA, USA; 254 nm light; power output is $100 \mu \mathrm{J} / \mathrm{cm}^{2}$; samples were placed in a 96-well plate; well area is $32 \mathrm{~cm}^{2}$; plate was placed $3 \mathrm{~cm}$ from power output source). Noncovalently bound deoxynucleotides were degraded by the addition of DNase I (0.1-4 U, $\left.37^{\circ} \mathrm{C}, 15-30 \mathrm{~min}\right)$. The reaction mixtures were then added to equal volumes of $2 \times$ loading buffer $(100 \mathrm{mM}$ Tris$\mathrm{HCl}, \mathrm{pH} 6.8,200 \mathrm{mM}$ dithiothreitol, $4 \%$ SDS, 2\% bromophenol blue, and $20 \%$ glycerol), boiled for $5 \mathrm{~min}$, and subjected to electrophoresis in an $8 \%$ polyacrylamide (acrylamide:bis-acrylamide, 29:1; supplemented with SDS) gel. Gels were transferred to nitrocellulose membranes prior to autoradiography $\left(-85^{\circ} \mathrm{C}\right.$ for $\left.30-72 \mathrm{~h}\right)$ or phosphorimager analysis with the Bio-Rad Molecular Imager system. Proteins are denatured and radioactively tagged, permitting accurate molecular weight determinations of the molecules recognized by EMSA.

\section{Immunoprecipitation of UV Cross- Linked DNA-Protein Complexes}

In some cases, following UV crosslinking of DNA-protein complexes and DNase I digestion, $1-2 \mu \mathrm{L}$ specific antibody were added to the reaction mixture and allowed to incubate overnight at $4^{\circ} \mathrm{C}$. PBS was added to the mixture to bring the final volume to $200 \mu \mathrm{L}$. Protein A slurry $(20-40 \mu \mathrm{L} ; 1: 1$ mix- ture of phosphate-buffered saline (PBS) and protein A-Sepharose ${ }^{\circledR}$ 4B beads; Sigma, St. Louis, MO, USA) was then added and allowed to mix for $2 \mathrm{~h}$ at $4^{\circ} \mathrm{C}$. The protein $\mathrm{A}$ beads were washed three times by gentle centrifugation in PBS and then resuspended in $6 \times$ loading buffer before heating at $100^{\circ} \mathrm{C}$ for $2 \mathrm{~min}$. DNA-protein complexes released from the protein $\mathrm{A}$ beads are denatured and loaded into SDS-polyacrylamide gels.

\section{Electrotransfer to Nitrocellulose Membranes and Immunoblot Procedures}

In some cases, following PAGE, proteins were electrotransferred onto nitrocellulose membranes (Magna NT; $0.22 \mu \mathrm{M}$ pore size; Micron Separation, Westborough, MA, USA). Following procedures described previously (11), blots were pre-soaked in $1 \times$ TBST $\left(0.05 \%\right.$ Tween ${ }^{\circledR} 20,20 \mathrm{mM}$ Tris- $\mathrm{HCl}$, $\mathrm{pH} \mathrm{7.5,500} \mathrm{mM} \mathrm{NaCl}$ ) and then blocked with $10 \%$ dried milk in $1 \times$ TBS (20 mM Tris- $\mathrm{HCl}, \mathrm{pH} 7.5,500$ $\mathrm{mM} \mathrm{NaCl}$ ) for $1 \mathrm{~h}$ before the addition of affinity-purified rabbit polyclonal antibody against the mouse GR $\alpha$ (Santa Cruz Biotechnology). The primary antibody was then incubated for $1 \mathrm{~h}$ at room temperature with gentle agitation, followed by successive membrane washes (4 times, $15 \mathrm{~min} /$ wash, room temperature). Goat anti-rabbit conjugated-horseradish peroxidase secondary antibody was diluted $(1: 10,000)$ in antibody buffer $(1.5 \mathrm{~mL}$ FCS +13.5 $\mathrm{mL} 1 \times \mathrm{TBS})$ and overlaid onto the blot for $1 \mathrm{~h}$ with gentle agitation. Membranes were then washed four times with $1 \times$ TBST and then developed using the SuperSignal ${ }^{\circledR}$ Western pico chemiluminescence substrate (Pierce Chemical, Rockford, IL, USA).

\section{RESULTS AND DISCUSSION}

\section{GREs in the Rat $\beta_{1}$-AR Gene}

Based on other promoter systems exhibiting negative glucocorticoid responsiveness, we conjectured that mammalian GR might not interact solely with the prototypical GRE motif (5'TGTTCT-3'), which in the $\beta_{1}$-AR gene 
consists of only one half-site and not the complete palindromic GRE. We endeavored to verify this hypothesis by using GRs expressed in eukaryotic systems, instead of in vitro synthesis systems, and using sources of GR that have undergone eukaryotic or mammalian-based posttranslational modifications. We have also developed and utilized a sensitive UV cross-linking/ immunoprecipitation assay to verify interaction of mammalian GR with the upstream region of the rat TAT gene, known to contain a palindromic GRE, and then tested this assay to validate GR interaction with upstream sequences from the rat $\beta_{1}$-AR gene.

\section{GR Expressed by Baculovirus Binds to the Consensus GRE but Not to the Half-Site Elements Contained in the Rat $\beta_{1}$-AR Gene}

Baculovirus-expressed GR was obtained from Affinity Bioreagents (kit RP500A; contains recombinant human GR expressed in insect cells) and was used in EMSA binding reactions with the consensus palindromic GRE probe. A high molecular weight complex was formed that was also supershifted in the presence of anti-GR $\alpha$ antibody (detects human, rat, and mouse GR $\alpha$; Figure 1). This same high molecular weight complex was not formed when mutant GRE probes, containing either a single obliterated half-site (upstream or downstream) or a completely obliterated site containing scrambled upstream and downstream half-sites, were used for EMSA (Figure 1). $\beta_{1}$-AR probes containing the sequences -970 to -920 and -2793 to -2743 , both subregions containing the GRE half-site $5^{\prime}$-TGTTCT3', were used for EMSA. These probes are composed of essentially identical subregions used by other investigators (9) in their examination of $\beta_{1}$-AR upstream sequence interaction with GR generated from reticulocyte lysates. No high molecular weight complexes were formed with our $\beta_{1}$-AR probes, demonstrating that the baculovirus-expressed GR was unable to recognize potential
GRE half-site targets in the $\beta_{1}$-AR gene or that additional flanking DNA was required for complete GR interaction. In addition, when anti-GR $\alpha$ Affinity Bioreagents antibody was added to the complexes containing the baculovirusexpressed GR and $\beta_{1}$-AR GRE halfsite probes, no antibody supershifts were formed, indicating that the background mobility shifts (Figure 2, see arrows) may have been due to nonspecific interaction with vector proteins extracted from the baculovirus expression recombinants.

In other EMSA experiments, we endeavored to use GRs obtained from mammalian CV-1 cells. Since baculovirus-expressed GRs undergo unique posttranslational modifications (e.g., Olinked glycosylation) not normally found in mammalian cells, we hypothesized that the absence of specific mobility shifts using the $\beta_{1}$-AR GRE half-site probes and baculovirus-expressed GR may have been the result of unique properties of the O-linked glycosylated GR molecule. Interestingly, CV-1 extracts yielded specific mobility shifts (Figure 2A; see arrows) when using the palindromic GRE probe, but not with the mutagenized GRE probe containing two ineffective half-sites with GT-to-CA substitutions in both half-sites. In addition, specific mobility shifts were not visualized using either of the two $\beta_{1}$-AR GRE half-site probes (-970 to -920 and -2793 to -2743) and the CV-1 extracts (Figure 2B). We hypothesize that GRs are expressed in this particular $\mathrm{CV}-1$ cell line and are available for in vitro interaction in an EMSA binding assay. No antibody supershifts, using either the Affinity Bioreagent or Santa Cruz Biotechnology antibodies recognizing GR, were detected using the palindromic GRE probe and CV-1 extracts (unpublished observations); since both commercially available antibodies have been characterized for use in antibody supershift experiments and both have been developed for antigenic reactivity to human GR, we speculate that the lack of antibody supershift in this experiment may be due to potential dissimilarities in antigen-
Figure 1. EMSA using baculovirus-expressed GR. Probes used in EMSA included the consensus palindromic GRE probe, mutant GRE probes containing obliterated single (upstream or downstream) or double half-sites (see mutant probe designations and descriptions in the Materials and Methods section), and $\beta_{1}$-AR probes containing the sequences -970 to -920 and -2773 to -2743 , both subregions containing the GRE half-site $5^{\prime}-$ TGTTCT- $3^{\prime}$. Supershift experiments used anti-GR $\alpha$ antibodies obtained from Affinity Bioreagents. Arrows indicate background mobility shifts potentially due to nonspecific interaction with vector proteins extracted from the baculovirus expression recombinants. 
antibody recognition of the monkey GR contained in CV-1 cells to the GR antibodies.

Thus, specific electrophoretic mobility shifts were not observed in EMSA using oligonucleotide probes containing the rat $\beta_{1}$-AR GRE half-sites and either baculovirus-generated recombinant human GR or mammalian GR obtained from monkey CV-1 cells.

\section{UV Cross-Linking/Immuno- precipitation Assay Indicates Binding of Mouse GR to the Rat TAT Fragment Containing a Palindromic GRE and to the Rat $\beta_{1}$-AR Fragments Containing Upstream Promoter Sequences}

We wanted to test the utility of an established cell line expressing mammalian GRs to recognize the palindromic GRE probe and $\beta_{1}$-AR GRE half-site probes. The mouse $3 \mathrm{~T} 3$ cell line has been characterized to express GRs using the Santa Cruz Biotechnology GR antibody in immunoblot analyses (as demonstrated in the company product literature) and was experimentally validated in our laboratory (Figure $3 \mathrm{~A}$, extreme left lanes). In spite of a great deal of published information concerning GRs, primarily using purified receptor preparations, there are limited commercially available antibody reagents recognizing GR. In addition, it has been difficult to confirm specific cell lines or tissues as being GR-deficient and to use these materials as verified negative controls for our experiments; some previously characterized cell lines (e.g., CV-1 cells) identified as GR-negative by functional criteria contain GR-immunoreactive protein identical in size to GR identified in the mouse $3 \mathrm{~T} 3$ cell line (see below).

A $100-\mathrm{kDa}$ molecule (major immunoreactive band) was visualized by immunoblot analyses using both 3T3 and $\mathrm{CV}-1$ extracts (Figure $3 \mathrm{~A}$ ); this is consistent with our positive EMSA analyses using CV-1 extracts and the palindromic GRE probe (Figure 2A). Both $3 \mathrm{~T} 3$ and $\mathrm{CV}-1$ extracts were used to validate the existence of a $100-\mathrm{kDa}$ molecule, immunoreactive to Santa Cruz Biotechnology GR antibodies, in both cell lines, and to help justify the use of CV-1 extracts as a source of GR for EMSA displayed in Figure 2. In addition, a slightly larger molecular weight component (minor immunoreactive band) of reduced band intensity was visualized in the $3 \mathrm{~T} 3$ lane (Figure $3 \mathrm{~A}$ ); faint traces of this minor immunoreactive component were also visualized in other immunoblots of $\mathrm{CV}-1$ extracts (unpublished observations). To develop an EMSA/UV cross-linking assay approach for GR-probe interaction, we synthesized PCR probes internally labeled with $[\alpha-32 \mathrm{P}] \mathrm{dCTP}$ and subjected DNA-protein complexes to UV cross-linking and DNase I digestion. Cell extracts or UV cross-linked EMSA reactions were denatured and electrophoresed in polyacrylamide gels, electrotransferred to nitrocellulose membranes, and subjected to immunoblot analysis using antibodies immunoreactive to the mouse GR (anti-GR Santa Cruz Biotechnology antibody; Figure $3 \mathrm{~A}$ ). When the internally labeled palindromic GRE probe and CV1 or 3 T 3 extracts were used in the
EMSA/UV cross-linking assay and subjected to phosphorimager analyses, we identified a predominant, radioactively tagged, cross-linked band, equivalent in molecular weight to the slightly larger immunoreactive GR component visualized by direct immunoblot analyses of cell extracts (Figure 3, B and C; see asterisks; these bands are equivalent in molecular weight to the minor immunoreactive band visualized in Figure 3A). The intensity of this predominant radioactive cross-linked band increases with higher DNase I doses (1-2 U), and all other higher molecular weight complexes observed at lower DNase I doses (0.1-0.5 U) become eliminated. The high molecular weight complexes observed at the top of the gel could represent radioactively tagged cross-linked DNA, which becomes eliminated with higher doses of DNase I. The $100-\mathrm{kDa}$ molecule is observed in all lanes in the immunoblot analyses (Figure 3A), including the UV cross-linked EMSA samples, and is due to the presence of nonradioactively

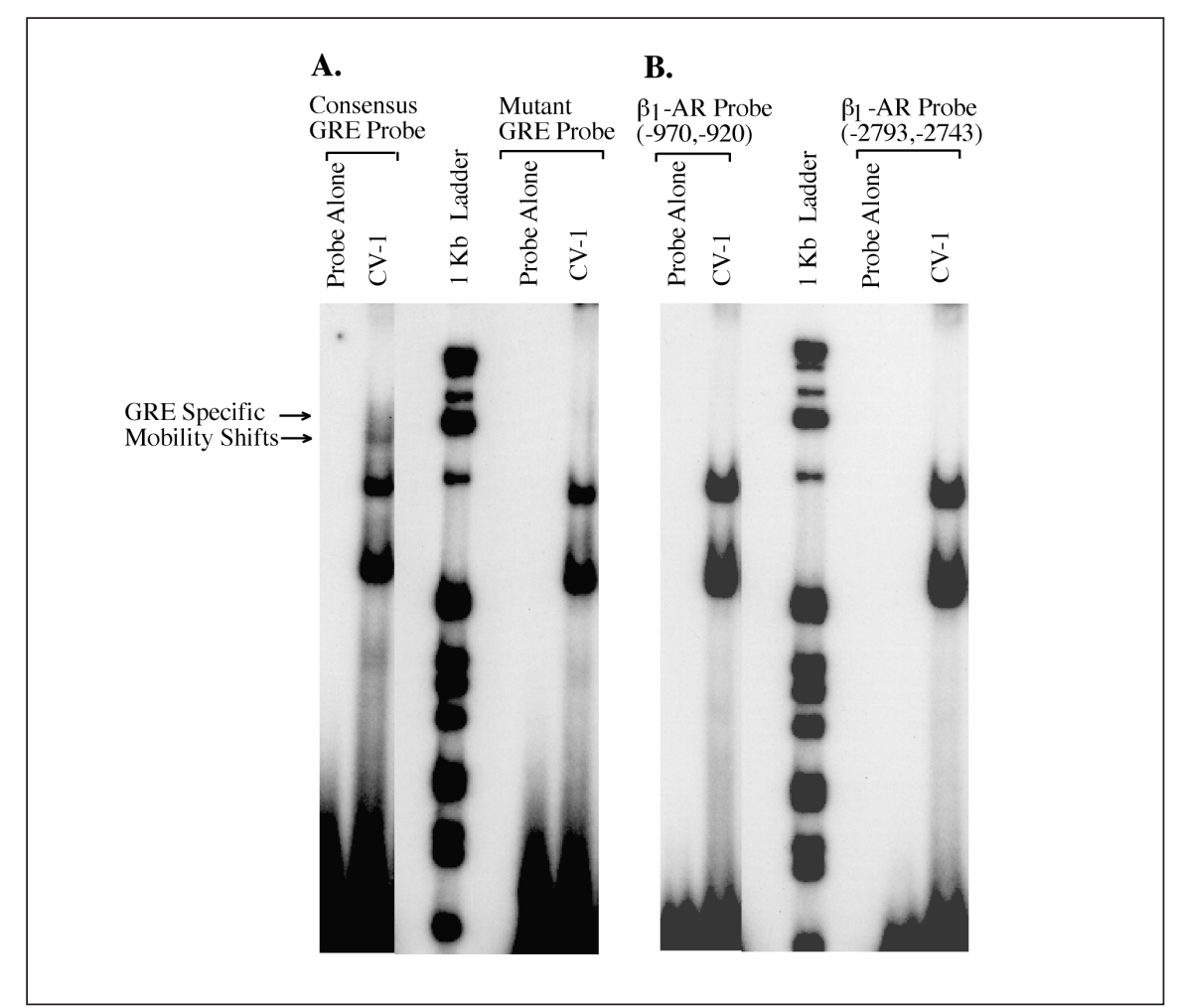

Figure 2. EMSA using CV-1 extracts. (A) EMSA using the consensus palindromic GRE probe and mutant GRE probe containing obliterated double half-sites (see Materials and Methods section for probe description). (B) EMSA using $\beta_{1}$-AR probes containing the sequences -970 to -920 and -2773 to -2743 , both subregions containing the GRE half-site 5'-TGTTCT-3'. Radioactively tagged 1-kb ladders are used for position referencing of DNA-protein complexes and are not used for the direct determination of size. 
tagged unbound GR molecules present in the original EMSA reaction. The anti-GR Affinity Bioreagents antibody, while recognizing baculovirus-expressed GR, was not able to recognize GR obtained from mouse 3T3 cells (unpublished observations) and was not used in any experiments requiring the detection of mammalian GR.

To test for the specificity of interaction of the mammalian GR with the internally labeled palindromic GRE probe, we conducted competition experiments using nonradioactive TAT GRE competitors (exact 524-bp sequence as the radioactive probe) and nonradioactive consensus or mutant GRE sequence competitors (20-bp dsDNAs containing the consensus GRE or mutagenized GRE) (Figure 4). We have also included a double-stranded, nonspecific competitor control that contains rat $\beta_{1}$-AR sequence -396 to -367 . This sequence, called GS-1, contains AP-2 consensus elements and interacts with a major protein of $117 \mathrm{kDa}$ and additional proteins of 37 and 35 $\mathrm{kDa}(12)$. All competitors were used in binding reactions utilizing the PCR- generated TAT GRE probe and 3T3 extracts in the UV cross-linking/immunoblot assay. As observed in Figure 4, we have successfully competed GR interaction with the TAT GRE probe using the homologous competitor [intensity values of 0.76 and 0.51 for $1 \times$ and $10 \times$ competition reactions, respectively (in top left panel), compared to a normalized value of 1.0 for probe-GR interaction alone], and the specific consensus GRE competitor (intensity values of 0.68 and 0.26 for $1 \times$ and $10 \times$ competition reactions, respectively, compared to a normalized value of 1.0 for probe-GR interaction alone). Competition with the latter competitor indicates that GR interaction may occur with the consensus GRE sequence plus some additional flanking DNA contained in the competitor sequence.

It is important to note, however, that competitions using higher concentrations $(10 \times$ molar excess) of the consensus GRE competitor, and to a lesser degree the homologous competitor as well, do not appear to compete reproducibly with the GR-TAT GRE probe interaction, occasionally yielding ra- dioactive band intensity levels approaching those observed in noncompetitor control reactions. At the higher concentrations of consensus GRE competitor, as is the case with all the oligonucleotide competitors used in this study, the DNase I to DNA substrate ratio becomes substantially skewed, altering the ability of the enzyme to uniformly digest the radioactive target DNA, compared to radioactive target DNAs in control reactions lacking competitor. In our experience, the amount of DNase I for a specific DNA mass must be titrated for optimal visualization of the radioactive, UV cross-linked GR band (Figure 3). We speculate that this may also be the explanation for the occasional observation of higher intensity UV crosslinked GR bands when using increasing concentrations $(10 \times$ molar excess $)$ of homologous DNA competitor. Retitration of DNase I may not be possible because of the need to equalize DNA mass among the different competition reactions using carrier DNA and the unexpected and variable signal quenching observed using traditional DNA carriers such as salmon sperm DNA (unpublished observations); we speculate that the carrier DNA may contain GR recognition sites, or perhaps nonspecific adsorption sites, and that the addition of varied amounts of carrier DNA to equalize mass results in variable radioactive signal quenching. In addition, in the case of the consensus GRE oligonucleotide competitions, we speculate that the interaction of GR to the TAT GRE probe is stronger, presumably because of multiple DNAprotein points of contact, than to the much shorter consensus GRE oligonucleotide competitor. Hence, the GR band intensity resulting from the consensus GRE oligonucleotide competition, unlike that observed with the homologous DNA competition, is more easily affected by the band intensity distortion resulting from the skewing of the DNase I to DNA substrate ratio. We illustrate the results shown in Figure 4 to demonstrate trends in the competitions, but with the caveat that especially in the case with the oligonucleotide competi- 
tors, only the low concentration ( $1 \times$ molar excess) competitor amounts would yield reproducible results.

As expected, no competition was observed using the GS-1 nonspecific competitor control, which does not contain consensus GRE sequences. In the one experiment displayed in Figure 4, no decrease in the radioactive intensity of the UV cross-linked GR molecule was observed when using either the $1 \times$ or $10 \times$ concentrations of GS-1 competitor (Figure 4 ; compare the $1 \times$ and $10 \times$ competition lanes; intensity values of $1.0+$ and $1.0+$ for the $1 \times$ and $10 \times$ competition reactions, respectively, compared to normalized value of 1.0 for the probe-GR interaction alone lane). In addition, as predicted, in the one experiment displayed in Figure 4, very limited or negligible competitions were observed when using the mutagenized GRE oligonucleotide containing two ineffective half-sites (Figure 4; compare the $1 \times$ and $10 \times$ competition lanes; intensity values of 0.81 and 0.84 for the $1 \times$ and $10 \times$ competitions, respectively, compared to normalized value of 1.0 for the probe alone lane). Repeat experiments $(n=3)$ of the $1 \times$ molar competition experiments displayed in Figure 4 yielded similar results; competitions using the homologous 524-bp competitor, specific consensus GRE competitor, GS-1 nonspecific competitor, and mutagenized GRE competitor, all conducted at $1 \times$ molar competitor excess in a single experiment, provided GR band intensity values of $0.79 \pm 0.06,0.79 \pm 0.07,1.0+$, and $1.0+$, respectively, compared to normalized values of 1.0 for the probe-GR interaction alone lanes ( \pm values are equivalent to 1 standard deviation). For reasons mentioned above, conducting competitions using the higher concentration $(10 \times$ molar excess $)$ of competitor yielded varied results and were not quantified in these repeat experiments.

All radioactively tagged GR molecules were verified by electrotransfer of proteins from gels to blots, with subsequent immunostaining of blots using the GR antibody (Santa Cruz Biotechnology); all radioactively tagged GR molecules were immunoreactive with the GR antibody in the immunoblot analyses (Figure 4).

We refined the UV cross-linking assay by conducting direct immunoprecip- itations of UV cross-linked DNA-protein complexes and utilized not only the internally labeled palindromic GRE probe derived from the TAT gene but also longer $\beta_{1}$-AR upstream and downstream probes containing the GRE halfsites and additional flanking DNA. In this experiment, UV cross-linked DNA/ 3T3 complexes were DNase I-treated
(2-4 U) before direct immunoprecipitation with anti-GR Santa Cruz Biotechnology antibodies. A higher DNase I dose was utilized in some cases (4 U) to ensure complete digestion. The 3T3 cell extracts and UV cross-linked, nonimmunoprecipitated DNA/3T3 complexes were also included in the electrophoretic analysis. Proteins were electrophoresed

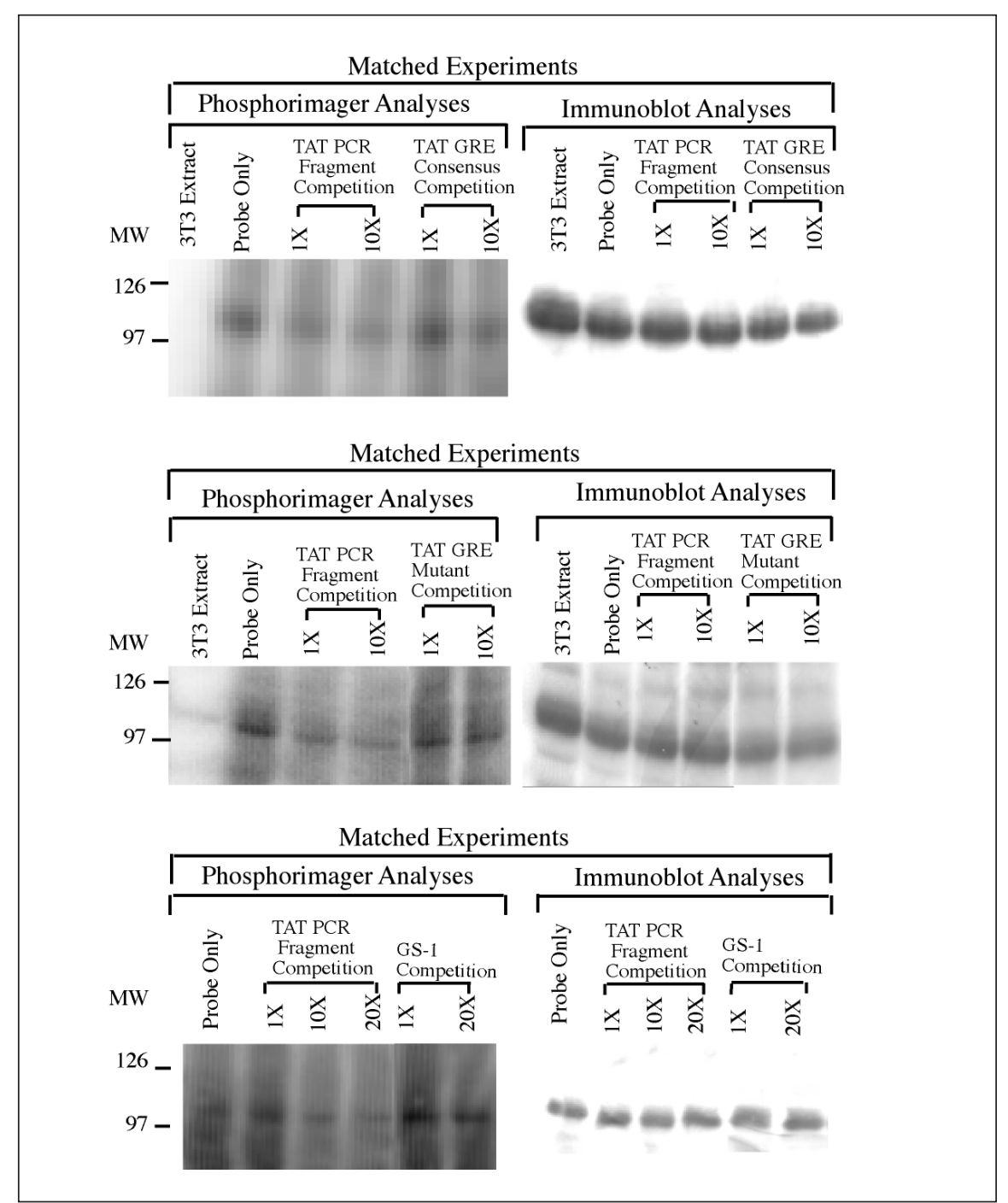

Figure 4. Competition assays using the internally labeled TAT GRE probe and 3T3 extracts in the UV cross-linking/immunoblot assay. Competitors include the 524-bp TAT GRE fragment (exact sequence as probe), nonradioactive consensus GRE sequence (20-bp double-stranded oligonucleotide containing the consensus GRE with additional flanking DNA), nonradioactive mutagenized GRE sequence [20-bp double-stranded oligonucleotide containing mutagenized GRE (two ineffective half-sites) plus additional flanking DNA], and the GS-1 nonspecific competitor sequence. Competitors were added at $1 \times$, $10 \times$, and/or $20 \times$ molar excess compared to the molar concentration of internally labeled TAT GRE probe. Signal intensities for each lane were quantitated using the Quantity One software program (Bio-Rad Laboratories), subtracted for background intensities in each lane, and then normalized against the band intensity for the control interaction without competitor within each individual gel. Note that GS-1 competitions and corresponding control interaction, as well as mutant GRE competitions and corresponding control interaction, were obtained from different regions of the same gel and have been electronically juxtaposed for this illustration. Phosphorimager signals are displayed in the left panels; all corresponding immunoblot analyses for each gel are displayed immediately adjacent in the right portion of the figure. 
in polyacrylamide gels and subsequently electrotransferred to nitrocellulose membranes for immunoblot analysis using the anti-GR Santa Cruz Biotechnology antibodies. This additional immunoblot step was conducted to assist in the alignment of the immunoprecipitated, radioactively tagged GR (Figure 5A, right subpanel) with molecules identified in the UV cross-linked complexes and with GR identified by direct immunoblot analysis of 3T3 extracts (Figure 5A, left subpanel). We observe that the immunoprecipitations conducted with the internally labeled palindromic GRE probe and the $\beta_{1}$-AR probe containing the upstream and downstream GRE half-sites recognize a GR-immunoreactive protein that co-migrates with GR identified by direct immunoblot analysis of 3T3 extracts (compare GR immunoreactive band and radioactively tagged GR band in Figure 5, A and B). Additional bands appearing in the immunoprecipitation lanes of the immunoblot analyses (Figure $5 \mathrm{~A}$; refer to bands in the $65-30 \mathrm{kDa}$ range) are due to immunoreactivity of the secondary antibodies in the immunoblot analysis reacting to the immunoglobulin molecules used in the initial immunoprecipitations. The $60-\mathrm{kDa}$ molecule in 3T3 extracts is sporadically observed (compare Figures 3 and 5) and was also observed in the Santa Cruz Biotechnology product literature. Note that the extreme right lane of the gel displayed in the left subpanel in Figure 5A (demonstrating immunoblot analysis of immunoprecipitated UV cross-linked GR bound to the $\beta_{1}$-AR $[-2844,-2142]$ probe) was inadvertently cropped just before blot transfer but after phosphorimager analysis, resulting in a truncated lane. The corresponding phosphorimage (Figure 5A, right subpanel) demonstrates the correct width of the lane. Repeat UV cross-linking/immunoprecipitation experiments were conducted using the internally labeled TAT GRE probe and higher amounts of anti-GR Santa Cruz Biotechnology antibodies in the immunoprecipitation reactions, resulting in an enhancement in signal intensity of the radioactively tagged immunoprecipitated GR (compare experiments displayed in Figure 5, A and B, using 1 and $3 \mu \mathrm{L}$ anti-GR Santa Cruz Biotechnology antibodies, respectively). Using the amplified $\beta_{1}$-AR probe containing the downstream GRE half-site and higher amounts of anti-GR Santa Cruz Biotechnology antibodies $(3 \mu \mathrm{L})$ in the immuno- precipitation reaction, we have also readily observed signals corresponding to the radioactively tagged immunoprecipitated GR (Figure 5B).

Using 30-bp double-stranded oligonucleotides containing the upstream $\beta_{1^{-}}$ AR GRE half-site (position -2767) or downsteam GRE half-site (position -945); we conducted competition reactions using the corresponding PCR-generated $\beta_{1}$-AR promoter fragment probes (703-bp upstream probe containing the GRE half-site at position -2767; 543-bp downstream probe containing the GRE half-site at position -945); and 3T3 extracts. Competitions using the 30-bp oligonucleotide duplexes were conducted to help identify the sites of GR interaction with the $\beta_{1}$-AR sequence. Multiple repeats of this competition experiment $(n=4)$ using the 30 -bp $\beta_{1}$-AR oligonucleotide duplexes demonstrated negligible competition, using a $1 \times$ molar competitor excess, between the $\beta_{1}$ AR promoter fragment probes and GR (phosphorimager signals were 1.0+ for all repeat competitions, compared to normalized value of 1.0 for the probeGR interaction alone lanes; $n=4$ experiments; unpublished observations). As expected, when homologous competi-

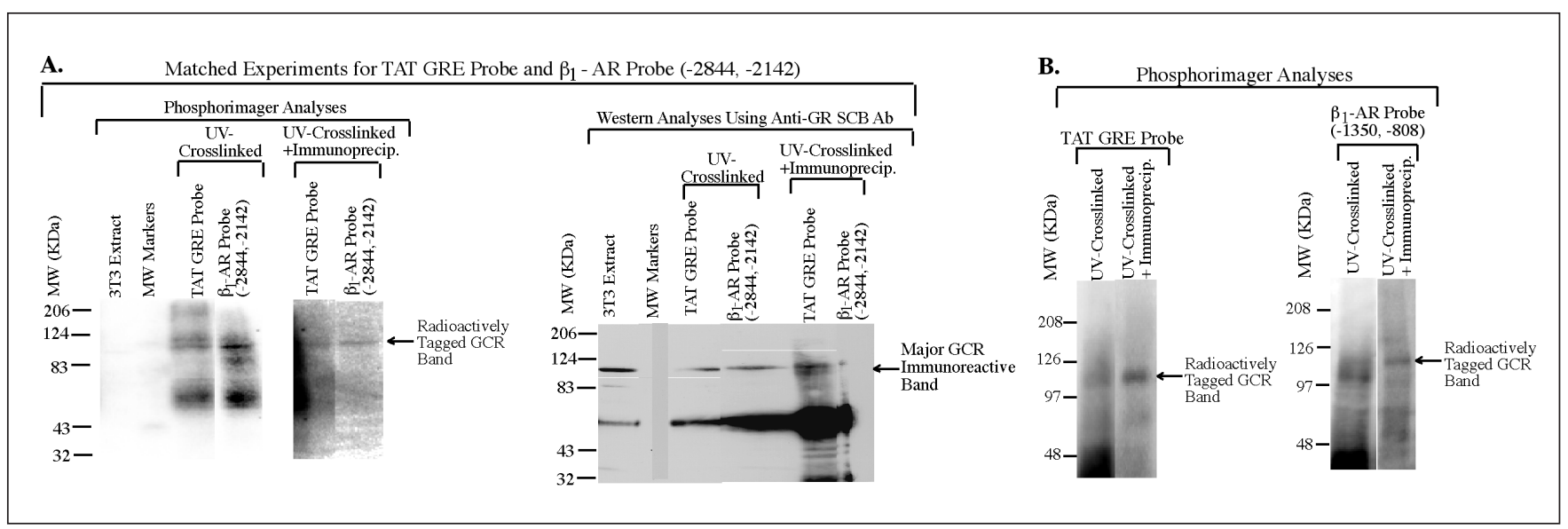

Figure 5. UV cross-linking/immunoprecipitation assays. (A) UV cross-linking/immunoprecipitation assays using the internally labeled palindromic GRE sequence amplified from the rat TAT gene and the amplified $\beta_{1}$-AR probe containing the upstream GRE half-site. The 3T3 extracts were used as binding material. UV cross-linking reactions were treated with DNase I (4 U) for $30 \mathrm{~min}$ at $37^{\circ} \mathrm{C}$; aliquots of these reactions were also immunoprecipitated with anti-GR Santa Cruz Biotechnology antibody $(1 \mu \mathrm{L})$. Proteins from UV cross-linking reactions and immunoprecipitation reactions were electrophoresed on a single polyacrylamide gel and subsequently electrotransferred for immunoblot analysis using the anti-GR Santa Cruz Biotechnology antibodies. This immunoblot analysis was conducted to confirm electrophoretic alignment of GR identified in 3T3 extracts (see extreme left lane in right subpanel of panel A) with the radioactively tagged GR identified in the immunoprecipitation reactions. Note that all UV cross-linked proteins are denatured and can be compared with both molecular weight size markers and with immunoreactive GR identified in the immunoblot analysis of 3T3 extract. Also note in the right subpanel that low-intensity phosphorimages of the UV cross-linking lanes have been juxtaposed with high-intensity phosphorimages of the immunoprecipitation lanes. (B, left subpanel) Repeat UV cross-linking/immunoprecipitation experiment using the internally labeled TAT GRE sequence, fresh radioisotope, and increased amounts of anti-GR Santa Cruz Biotechnology antibody (3 $\mu \mathrm{L}$ ). Note the enhancement of signal intensity of the radioactively tagged immunoprecipitated GR. (B, right subpanel) UV cross-linking/immunoprecipitation experiment using the amplified $\beta_{1}$-AR probe containing the downstream GRE half-site. The experiment was conducted as described in panel A, with the exception that fresh radioisotope was used and increasing amounts of anti-GR Santa Cruz Biotechnology antibody were applied in the immunoprecipitations. 
tors were used (exact 703-bp upstream and exact 543-bp downsteam nonradioactive competitors), successful competitions were observed (at $1 \times$ molar competitor excess: phosphorimager signals for the 703-bp upstream and 543bp downstream homologous competitors were $0.84 \pm 0.09$ and $0.80 \pm 0.09$, respectively, compared to normalized values of 1.0 for the probe-GR interaction alone lanes; $n=4$ experiments). In addition, when using nonspecific GS-1 competitors $(1 \times$ molar competitor excess), no decrease in the radioactive intensity of the UV cross-linked GR molecule was observed; phosphorimager signals were $1.0+$ for both the 703-bp upstream and 543-bp downstream $\beta_{1}$ AR promoter fragment probes, compared to normalized values of 1.0 for the probe-GR interaction alone lanes; $n$ $=4$ experiments; unpublished observations). For all gels, immunoblots were prepared and stained using GR antibodies to identify the exact position of the UV cross-linked band that represented GR. These experiments provide evidence that rat $\beta_{1}$-AR gene sequences recognize mammalian GRs expressed in mouse 3T3 cells and that the site of GR interaction is complex and does not appear to specifically contain the GRE half-sites.

These results are not surprising, in that negative GREs, or elements recognized for glucocorticoid-induced transcriptional repression, do not typically comprise GRE half-site sequences. Negative GREs are more diverse in sequence $(13,14)$; the negative GRE defined in the rat proopiomelanocortin promoter comprises the sequence $5^{\prime}$ GGAAGGTCACGTCCA-3' (13). In addition, as defined by DNase footprinting analyses, GR binds to the rat vasointestinal peptide receptor promoter at sequences unique to either the prototypical GRE or other known negative GREs (14).

This manuscript describes a sensitive UV cross-linking/immunoprecipitation assay for the detection of mammalian GR interaction with $\beta_{1}$-AR sequences. This assay was validated with the use of probes containing the palindromic GRE site in the rat TAT gene and experimentally demonstrates GR interaction with rat $\beta_{1}$-AR upstream sequences. With the use of short double-stranded oligonucleotide competitors, it appears that GR interaction with the rat $\beta_{1}$-AR upstream sequences is complex and does not directly recognize the GRE half-sites. However, we cannot preclude the possibility that other protein factors may recognize the $\beta_{1}$ AR GRE half-sites or surrounding sequences to form larger transcription complexes and complete the transactivation of the corresponding promoter.

\section{ACKNOWLEDGMENTS}

In addition to Y.B., P.K. contributed substantially to this manuscript. Special thanks are extended to Matthew Todd for his assistance in the development of the extended $\beta_{1}$-AR GRE recombinants. We also thank Crystal Daniels for support during earlier stages of this project. I also thank Dr. Tom Shearer for his overall support of the program. We acknowledge the help of Yibing Jia and Kalama Taylor of the Oregon National Primate Research Center Molecular Biology and Cell Culture Core Facilities, respective$l y$, for their assistance in automated DNA sequencing and cell culture propagation. P.K. is currently in the General Clinical Research Center at the Oregon Health \& Science University. X.L. is now in the Department of Medical and Molecular Genetics at the Oregon Health \& Science University. This research was supported by National Institutes of Health grant nos. HL 42358 and $M H$ 63137, and by the American Heart Association (AHA) grant no. $0250017 N$. C.A.M. was also a former recipient of an AHA Established Investigatorship.

\section{REFERENCES}

1.Collins, S., M.G. Caron, and R.J. Lefkowitz. 1989. $\beta_{2}$-adrenergic receptors in hamster smooth muscle cells are transcriptionally regulated by glucocorticoids. J. Biol. Chem. 263:9067-9070.

2.Collins, S., M.J. Lohse, B. O'Dowd, M.G. Caron, and R.J. Lefkowitz. 1991. Structure and regulation of $\mathrm{G}$ protein-coupled receptors: the $\beta_{2}$-adrenergic receptor as a model. Vitam. Horm. 46:1-39.

3.Kiely, J., J.R. Hadcock, S.W. Bahouth, and C.C. Malbon. 1994. Glucocorticoids downregulate $\beta_{1}$-adrenergic receptor expression by suppressing transcription of the receptor gene. Biochem. J. 302:397-403.

4.Fève, B., L.J. Emorine, M.-M. Briend-
Sutren, F. Lasnier, A.D. Strosberg, and J. Pairault. 1990. Differential regulation of $\beta_{1}$ and $\beta_{2}$-adrenergic receptor protein and mRNA levels by glucocorticoids during 3T3-F442A adipose differentiation. J. Biol. Chem. 265:16343-16349.

5.Guest, S.J., J.R. Hadcock, D.C. Watkins, and C.C. Malbon. 1990. $\beta_{1}$ - and $\beta_{2}$-adrenergic receptor expression in differentiating $3 \mathrm{~T} 3$ $\mathrm{L} 1$ cells. Independent regulation at the level of mRNA. J. Biol. Chem. 265:5370-5375.

6.Collins, S., M.A. Bolanowski, M.G. Caron, and R.J. Lefkowitz. 1989. Genetic regulation of $\beta$-adrenergic receptors. Annu. Rev. Physiol. 51:203-215.

7.Miner, J.N., M.I. Diamond, and K.R. Yamamoto. 1991. Joints in the regulatory lattice: composite regulation by the steroid receptorAP1 complexes. Cell Growth Differ. 2:525530.

8.Cornett, L.E., F.C. Hiller, S.E. Jacobi, W. Cao, and D.W. McGraw. 1998. Identification of a glucocorticoid response element in the rat $\beta_{2}$-adrenergic receptor gene. Mol. Pharm. 54:1016-1023.

9.Bahouth, S.W., E.A. Park, M. Beauchamp, X. Cui, and C.C. Malbon. 1996. Identification of glucocorticoid repressor domain in the rat $\beta_{1}$-adrenergic receptor gene. Receptors Transduction 6:141-149.

10.Tseng, Y.-T., J.P. Stabila, T.T. Nguyen, B.G McGonnigal, J.A. Waschek, and J.F. Padbury. 2001. A novel glucocorticoid regulatory unit mediates the hormone responsiveness of rat $\beta_{1}$-adrenergic receptor gene. Mol. Cell Endo. 181:165-178

11.Kirigiti, P. and C.A. Machida. 1999. Electrophoretic mobility shift assay for detection of DNA binding proteins recognizing $\beta$ adrenergic receptor gene sequences, p. 431451. In C.A. Machida (Ed.), Adrenergic Receptor Protocols. Humana Press, Totowa, NJ.

12.Kirigiti, P., Y.-F. Yang, X. Li, B. Li, C.N. Midson, and C.A. Machida. 2000. Rat $\beta_{1}$ adrenergic receptor regulatory region containing consensus AP-2 element recognizes novel transactivator proteins. Mol. Cell Biol. Res. Comm. 3:181-192.

13.Drouin, J., Y.L. Sun, M. Chamberland, Y. Gauthier, A. De Lean, M. Nemer, and T.J. Schmidt. 1993. Novel glucocorticoid receptor complex with DNA element of the hormonerepressed POMC gene. EMBO J. 12:145-156.

14.Pei, L. 1996. Identification of a negative glucocorticoid response element in the rat type 1 vasoactive intestinal polypeptide receptor gene. J. Biol. Chem. 271:20879-20884.

Received 3 September 2002; accepted 10 April 2003.

Address correspondence to:

Curtis A. Machida

Department of Integrative Biosciences

School of Dentistry

Oregon Health \& Science University

611 SW Campus Drive

Portland, OR 97239, USA

e-mail:machidac@ohsu.edu 